\title{
Different Synthetic Routes of Group Six (VIB) Nano-Metal Borides
}

\section{Al-Zain OA* and Al-Masoudi RS}

Chemistry Department, Faculty of Science, King Abdulaziz University, Jiddah, Saudi Arabia

\begin{abstract}
For the first time, the synthetic comparison between the solid and liquid phases of metal borides was subjected to empirical investigation. The magnesiothermic reaction of $\mathrm{CrO}_{3}, \mathrm{~B}_{2} \mathrm{O}_{3}$, and $\mathrm{Mg}$ yielded the only $\mathrm{Cr}_{2} \mathrm{O}_{3}$. Similarly, the direct reaction of $\mathrm{CrO}_{3}$ or $\mathrm{CrCl}_{3}$ with elemental boron yielded chromium oxides. On the other hand, direct reaction of pure $\mathrm{Cr}$ metal with elemental boron in 1:1,1:2 and 1:3 ratios, showed that only 1:3 ratio, produced a mixture of $\mathrm{Cr}_{2} \mathrm{~B}, \mathrm{Cr}_{2} \mathrm{O}_{3}$, and $\mathrm{CrO}_{3}$ (minimal oxides). Going down group six, (VIB) $\mathrm{MoO}_{3}$ and $\mathrm{WO}_{3}$, reacted directly with elemental boron in a muffle furnace at 500,700 and $1000^{\circ} \mathrm{C}$ for 2,2 and 15 hours respectively, yielding black beautiful nanocrystals of Mo $\mathrm{B}_{4}, \mathrm{MoB}$, $\mathrm{WB}_{4}$, and $\mathrm{W}_{2} \mathrm{~B}_{5}$. The liquid phase reaction of molybdenum pentachloride, $\mathrm{MoCl}_{5}$, with sodium borohydride, $\mathrm{NaBH}_{4}$ (1:5 ratio), produced black nanocrystals and nanorods of $\mathrm{MoB}$ and $\mathrm{MoB}_{4}$. Reacting chromium trichloride with sodium borohydride, $\mathrm{NaBH}_{4}$ (1:3 ratio), in solution, produced pure black nanocrystals and nanorods of $\mathrm{Cr}_{2} \mathrm{~B}$, while the reaction of chromium dichloride with sodium borohydride, $\mathrm{NaBH}_{4}\left(1: 2\right.$ ratio), in solution, produced a mixture of $\mathrm{Cr}_{2} \mathrm{~B}$ and $\mathrm{Cr}_{2} \mathrm{O}_{3}$.
\end{abstract}

Keywords: Nanocomponds; Metal Borides; Nanowires; Nanocrystals

\section{Introduction}

A great number of transition metal borides were synthesized. That is due to their special characters including high hardness, high melting points, high-temperature strength, corrosion resistance, chemical stability, wear resistance and electrical properties [1-6]. Several chromium borides are known bytheir wide range of atomic ratios $\left(\mathrm{Cr}_{5} \mathrm{~B}_{3}, \mathrm{CrB}, \mathrm{Cr}_{3} \mathrm{~B}_{4}, \mathrm{Cr}_{2} \mathrm{~B}_{3}\right.$ and $\mathrm{CrB}_{2}$ [7-9]. Two among these, $\mathrm{CrB}$ and $\mathrm{CrB}_{2}$, are more important since they are closer to the properties mentioned above. Synthetic preparation of chromium boride is a very difficult process, because binary side products are always there including chromium oxides. Several synthetic methods have been tried like combustion synthesis [8,9], thermal evaporation process [10], and pulsed magnetron sputtering [11].

On the other hand, molybdenum and tungsten borides have similar properties to those of chromium borides, except solubility of the oxides in water where Molybdenum is sparingly soluble while tungsten is insoluble. Molybdenum forms $\mathrm{MoB}, \mathrm{Mo}_{2} \mathrm{~B}, \mathrm{MoB}_{2}, \mathrm{Mo}_{2} \mathrm{~B}_{5}$ and $\mathrm{MoB}_{4}$ which synthesized through different methods, such as mechanochemical [12], electrochemical [13,14], and hydrothermal methods [15], self-propagating high-temperature synthesis, with highly exothermic reaction $[16,17]$. Tungsten forms $\mathrm{W}_{2} \mathrm{~B}, \mathrm{~W}_{2} \mathrm{~B}_{5}, \mathrm{WB}$, $\mathrm{WB}_{4}$, and $\mathrm{WB}_{12}$, which are prepared by many different methods such as chemical vapor deposition, solid-state reaction, ionic melts, selfpropagating high-temperature synthesis, arc plasma melting and mechanochemical method [18-21].

Even though, as mention above, several synthetic methods were applied to produce borides, almost none of these studies proved effective in the formation of single phase borides, rather a mixture of boride phases was formed $[22,23]$. In this study, two different methods were carried out in the synthetic processing of chromium, molybdenum and tungsten borides, namely, solid states and wet reaction.

\section{Experimentation/Empirical Analysis}

\section{Reagents}

Unless otherwise specified, reagent grade chemicals were employed. The precursor materials were Chromium trioxide (CrO3, $\mathrm{BDH})$, Molybdenum (VI) Oxide ( $\mathrm{MoO}_{3}$, Alfa Aesar), and Tungsten Oxide (WO3, Alfa Aesar). Boron Powder (Amorphous) (B, Loba Chemie),
Boron (III) Oxide $\left(\mathrm{B}_{2} \mathrm{O}_{3}\right.$, Alfa Aesar). Magnesium Powder (BDH). Sodium Borohydride (NaBH4, BDH), Chromium(III) Chloride (Anhydrous) $\left(\mathrm{CrCl}_{3}\right.$, Fluka AG, Chem), Chromium(III) Chloride Hexahydrate $\left(\mathrm{CrCl}_{3} \cdot 6 \mathrm{H}_{2} \mathrm{O}, \mathrm{BDF}\right)$, and Hydrochloric acid ( $\mathrm{HCl}$, SigmaAldrich, fuming $>37 \%$ ). Chromium Powder (Cr, Alfa Aesar).

The Powder X-ray diffraction (XRD) measurement was carried out with a Bruker D8 Advance diffractometer (CuKa $\lambda=1.54 \AA$ A; Ni filter; 40 $\mathrm{KV}, 40 \mathrm{~mA}$; Divergence slit: $1 \mathrm{~mm}$, LynxEye one-dimensional detector, Detector slit: $8 \mathrm{~mm}$ ). Scanning Electron Microscope and Transmission Electron Microscope (SEM and TEM) were performed by employing a microscope of model Titan 80-300 CT from FEI Company (Hillsboro, OR), which was equipped with a field emission gun (FEG) and a charged-Couple Devices (CCD) camera of $4 \mathrm{k} \times 4 \mathrm{k}$ pixels. Furthermore, the analyses of all samples were performed by operating the microscope at $300 \mathrm{kV}$ accelerating voltage in order to take full advantage of the highest spatial resolution possible from this microscope. An aperture of 100 microns was also inserted into the back-focal plane (BPF) of the objective lens, to improve the image contrast. The Magnetization measurement was carried out in an ever-cool Squid-Vibrating Sample Magnetometer (SVSM), from the manufacturer Quantum Design, USA.

\section{Synthesis}

\section{Solid phase}

Preparation of metal borides using boron oxide and magnesium

Chromium boride

$$
2 \mathrm{CrO}_{3}+\mathrm{B}_{2} \mathrm{O}_{3}+6 \mathrm{Mg} \stackrel{\Delta}{\longrightarrow} \mathrm{Cr}_{2} \mathrm{O}_{3}+2 \mathrm{~B}+6 \mathrm{MgO}
$$

*Corresponding author: Omar Al-Zain, Chemistry Department, Faculty of Science, King Abdulaziz University, Jiddah, Saudi Arabia, Tel: 966555600872 ; E-mail: alzain2005@yahoo.com

Received: October 06, 2017; Accepted: October 14, 2017; Published: October 20, 2017

Citation: Al-Zain OA, Al-Masoudi RS (2017) Different Synthetic Routes of Group Six (VIB) Nano-Metal Borides. J Nanomed Nanotechnol 8: 464 doi: 10.4172/2157 7439.1000464

Copyright: (c) 2017 Al-Zain OA, et al. This is an open-access article distributed under the terms of the Creative Commons Attribution License, which permits unrestricted use, distribution, and reproduction in any medium, provided the original author and source are credited. 